\title{
HIGHLY LOCALIZED AZIMUTHAL MEASUREMENTS IN THE CROCUS REACTOR TOWARDS THE VALIDATION OF HIGH-FIDELITY NEUTRONICS CODES
}

\author{
Fanny Vitullo ${ }^{1}$, Vincent Lamirand ${ }^{1}$, Pavel Frajtag ${ }^{1}$, Gregory Perret ${ }^{1}$ and Andreas Pautz ${ }^{1,2}$ \\ ${ }^{1}$ École polytechnique fédérale de Lausanne (EPFL), 1015 Lausanne, Switzerland \\ ${ }^{2}$ Paul Scherrer Institut (PSI), 5232 Villigen PSI, Switzerland \\ Email: fanny.vitullo@epfl.ch, vincent.lamirand@epfl.ch
}

\begin{abstract}
Highly localized in-core measurements are necessary for the validation of neutron transport calculations with high spatial resolution. In the present work, a miniature neutron detector developed at EPFL in collaboration with PSI was used to carry out a set of thermal neutrons counting measurements in the zero-power CROCUS reactor core within a spatial range in order of $\mathrm{mm}$. The miniature detector, positioned close to the core reflector, shows a gradient of $+(4.29 \pm 0.10) \%$ in the count rate profile in the radial direction within $1.3 \mathrm{~cm}$, with higher values pointing towards the core reflector because of the higher share of neutrons in the thermal range. On the contrary, in a control rod guide tube the count rate gradient is $-(4.37 \pm 0.10) \%$ and it is directed towards the core center. The measured values are compared with the azimuthal trend of the normalized ${ }^{6} \mathrm{Li}$ reaction rate calculated with an iterative threesteps method performed with the Monte Carlo code Serpent 2. These measurements proved the feasibility of resolving spatial effects in the mm-range and they represent a basis for further investigating highly spatially-resolved phenomena in the CROCUS core.
\end{abstract}

KEYWORDS: azimuthal flux measurements, CROCUS zero-power research reactor, micro-reactor physics, miniature neutron detector.

\section{INTRODUCTION}

The increasing complexity of nuclear reactor concepts has been driven by the continuous endeavor to improve fuel performances and utilization in view of cost optimization by nuclear power plant operators. Modern light water reactors (LWRs) core designs present heterogeneities at multiple levels: from mixed core arrangements [1] to heterogeneous boiling water reactors (BWRs) fuel assemblies with one or more water channels and partial length fuel rods (e.g. the latest Westinghouse TRITON11 fuel assemblies [2]). The presence of such heterogeneities requires the precise knowledge of the spatial distribution of the neutron population in the core for both safety reasons and performances optimization. The local neutron flux can be predicted by means of neutron transport simulations with high spatial resolution. Although stochastic Monte Carlo represents as of today the standard for the evaluation of highly spatially-resolved neutron fluxes, the recent developments in deterministic neutronics codes are moving towards finer resolutions in space. When using deterministic neutronics codes for the prediction of localized effect in a reactor core, the code high-resolution capabilities must be validated against highly localized experimental results. However, the challenges to be faced in order to perform in-core and within-pin experiments are 
numerous. The accessibility to locations of interest, e.g. within the fuel lattice or inside fuel pins, remains the main limitation to carry out highly localized measurements in reactor cores of operating nuclear power plants. In order to face this issue, high-fidelity codes can be validated against experiments performed in zero-power reactors, having a better flexibility in operation, granting an easier access to the fuel lattice and allowing to evaluate neutronics effects without the influence of feedbacks related to thermal and fuel composition effects. In the past, radial and azimuthal fission cross sections have been measured within a fuel pin of a Westinghouse SVEA-96 Optima2 BWR assembly installed in the zero-power reactor PROTEUS [3] at Paul Scherrer Institut (PSI). The goal was to measure within-pin space-dependent fission power maps and actinide concentration profiles and to compare the measured values with the results of both deterministic and stochastic codes. Despite the success of this experiment, the employed neutron activation technique represents a limit whether the localized phenomena want to be observed in an online manner during dynamic neutronics events [4],[5]. Detection techniques with both a spatial resolution in the order of the millimeter and a good efficiency are a paramount to investigate local static and dynamic effects in zero-power reactors. In addition, a good insensitivity to the gamma radiation field present in the core and a high flexibility are desirable characteristics for such measuring devices.

In this framework, an innovative miniature neutron detector [6],[7] developed at the École polytechnique fédérale de Lausanne (EPFL) in collaboration with PSI was used to investigate highly spatially-resolved neutronics effects in the zero-power CROCUS reactor operated at EPFL. The goal of this work is to present a first set of highly localized measurements in the CROCUS reactor and compare the experimental data against Serpent 2 Monte Carlo simulations. These measurements pave the way for further investigation of localized phenomena in the CROCUS core and represent a basis for the validation of the high-fidelity capabilities of deterministic codes. In particular, the aims of the current work are to:

- Measure the azimuthal distribution of the neutron count rate within a control rod guide tube of CROCUS, made of the same aluminum cladding of the CROCUS fuel and used as an experimental channel in air, positioned at two different core locations.

- Compare the relative measurement data with the ${ }^{6} \mathrm{Li}$ normalized reaction rate calculated via the Monte Carlo code Serpent 2.

The present article is structured as follows. First, the miniature neutron detector is briefly described. Then, the CROCUS reactor and the experimental setup installed in the core are presented. In the third section, the experimental results are presented and in the last section they are compared with the Serpent 2 estimations.

\section{EXPERIMENTAL SETUP}

\subsection{Miniature neutron detector}

An advanced miniature neutron detector was developed and tested as of December 2017 at the Laboratory for Reactor Physics and System Behaviour (LRS) of EPFL in collaboration with the Laboratory for Particle Physics (LTP) at PSI. The detector sensitive volume of less than a $\mathrm{mm}^{3}\left(1 \mathrm{~mm}^{2}\right.$ surface and $0.2 \mathrm{~mm}$ thickness) consists of a screen composed by a mixture of zinc-sulphide ( $\mathrm{ZnS}$ ) inorganic scintillator and molecules of lithium- 6 fluoride $\left({ }^{6} \mathrm{LiF}\right)$, the latter acting as a converter of neutrons in the thermal energy range. The $\mathrm{ZnS}:{ }^{6} \mathrm{LiF}$ screen, visible in Fig. 1(a), is coupled to a silicon photon multiplier (SiPM) through a $10-\mathrm{m}$ optical fiber with a 3-mm external diameter. The optical fiber transports the light from the screen to the SiPM. The coupling between the $\mathrm{ZnS}:{ }^{6} \mathrm{LiF}$ screen and the optical fiber is completed by using an aluminum cap taped to the fiber outer jacket (see Fig. 1(b)). In the development version employed in the current set of experiments, the SiPM output signal is read by an analog read-out system designed to operate the detector in pulse mode. The train of light pulses originating from the scintillation process after a neutron interaction is translated into a square pulse that is used to count single neutron events. Figure 2 shows a complete schematic of the measuring system. A comprehensive description of the miniature detector and its associated electronics can be found in [6] and [7]. 


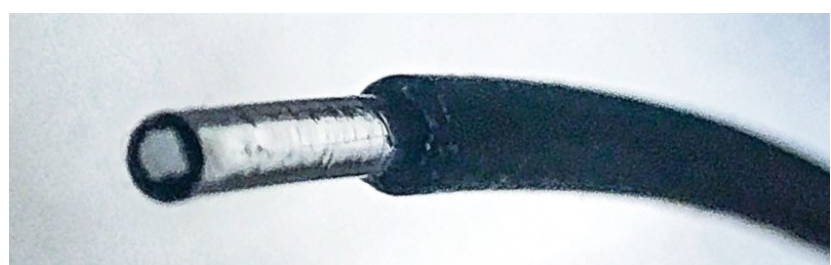

(a)

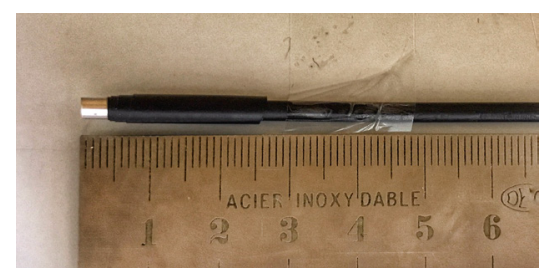

(b)

Figure 1. (a) Optical fiber front-end with $\mathrm{ZnS}:{ }^{6} \mathrm{LiF}$ screen visible at the fiber tip. (b) Picture of the miniature detector front-end.

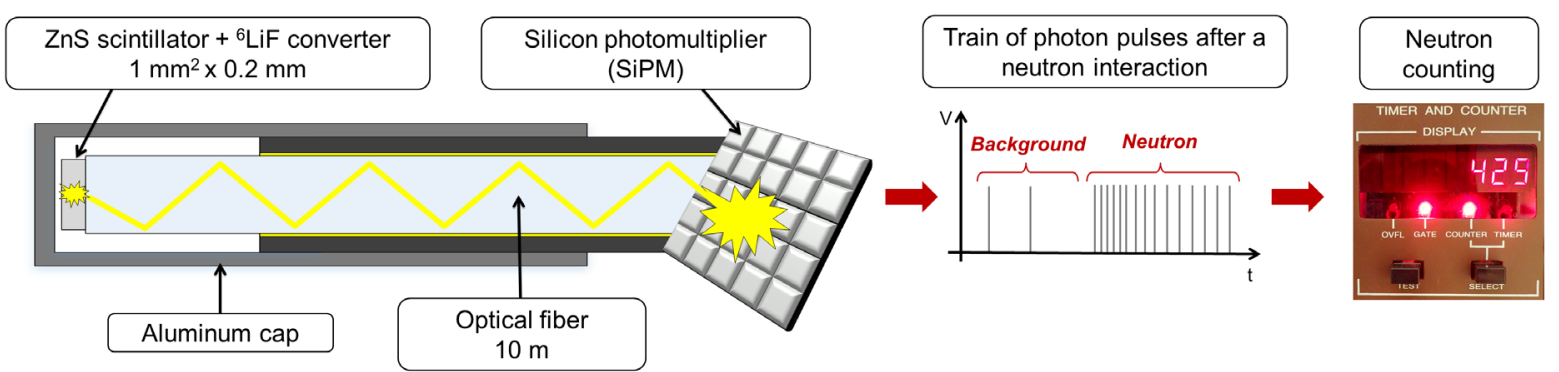

Figure 2. Schematic of the detection system, from the $\mathrm{ZnS}:{ }^{6} \mathrm{LiF}$ screen to the read-out electronics.

The main advantages of using this detector over other standard instrumentations consists in the miniature dimensions of its sensitive area that allow to perform localized measurement with a mm resolution and a minimum perturbation of the neutron. In addition, the detector has a good flexibility in positioning by means of simple tools and it is easily manufactured at a reduced price. The performances, assessed in [6] and [7], show a sensitivity of $(3.53 \pm 0.11) \times 10^{-4} \mathrm{cps} / \mathrm{n}$ to the total neutron flux when tested in the zero-power CROCUS reactor (described in Section 2.2) and a linear response up to a neutron flux of $6.2 \times 10^{7} \mathrm{~cm}^{-2} \cdot \mathrm{s}^{-1}$. The detector gamma-rejection capabilities were proven in [7].

\subsection{Setup in the CROCUS reactor}

The CROCUS reactor [8] is an experimental zero-power reactor operated at LRS, dedicated to teaching and research. The reactor core, being $1 \mathrm{~m}$ high and about $60 \mathrm{~cm}$ in diameter, is fueled with low enriched uranium and moderated with demineralized light water. Two different fuels are loaded in the core: an inner uranium oxide region with 336 rods enriched to $1.806 \%$ and an outer uranium metal region with 172 to 176 rods enriched to $0.947 \%$. The allowed fission power is limited to $100 \mathrm{~W}$ and its level is controlled either by adjusting the core water level or by inserting two $\mathrm{B}_{4} \mathrm{C}$ absorber control rods. Six independent safety systems are in place, including two cruciform safety blades and four water expansion tanks, each of which ensure a safe shut down in less than a second. The maximum achievable total neutron flux in CROCUS is $2.5 \times 10^{9} \mathrm{~cm}^{-2} \cdot \mathrm{s}^{-1}$ in the core center. The monitoring of the neutron population in CROCUS is performed by four ex-core detectors: two Photonis CFUD2 $1{ }^{235} \mathrm{U}$ fission chambers working in pulse mode and two Merlin Gerin $\mathrm{CC} 54{ }^{10} \mathrm{~B}$-coated compensated ionization chambers working in current mode. The four detectors, called "monitors" and visible in Fig. 3(a), are located in the core periphery into four detection channels attached to the upper grid plate. The slightly heterogeneous configuration due to the double fuel composition and the presence of a wide water reflector represent the distinct features of the CROCUS reactor. These heterogeneities might be of great interest for the validation of highly spatially-resolved nuclear codes. Moreover, the low technological uncertainties in the CROCUS design make this core a good reference for localized experimental measurements. 


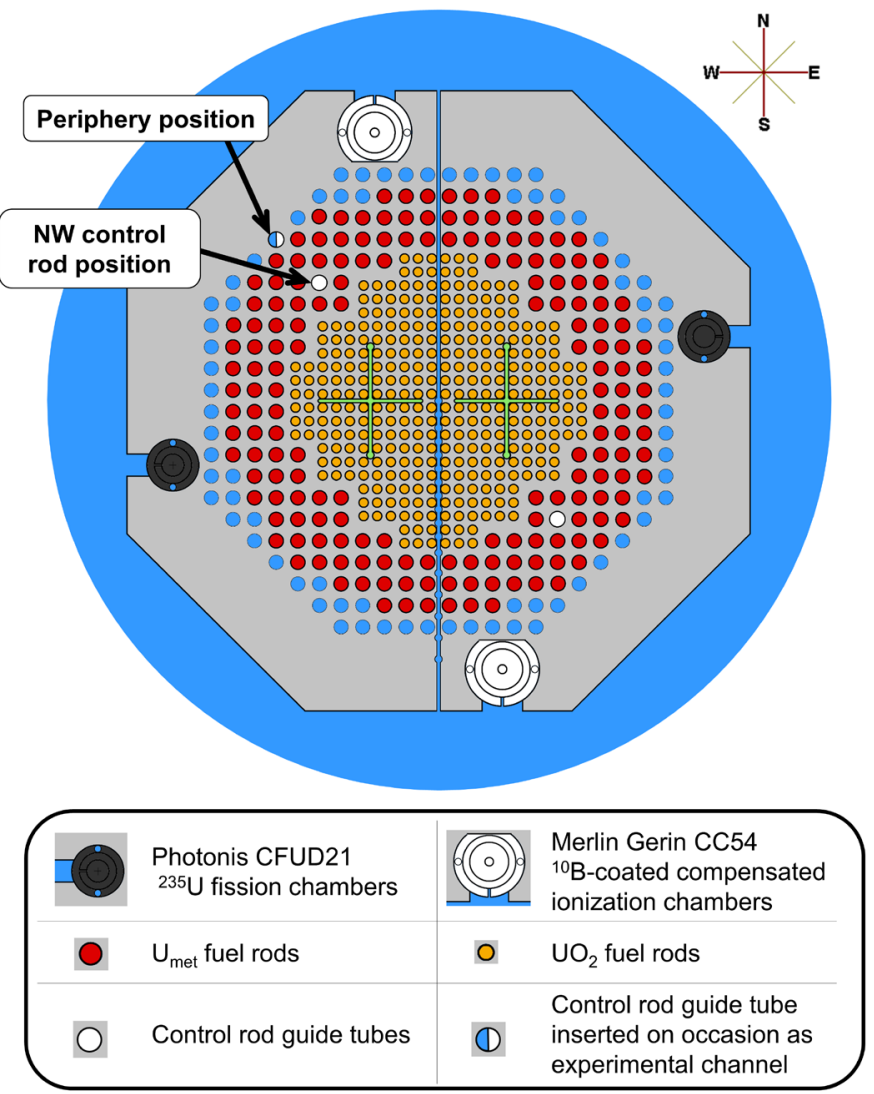

(a)

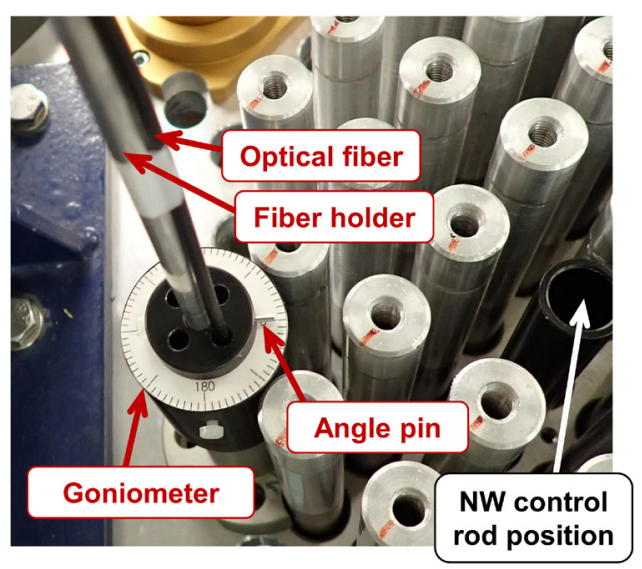

(b)

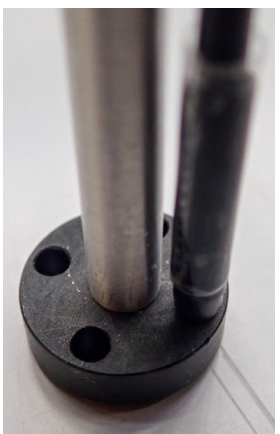

(c)

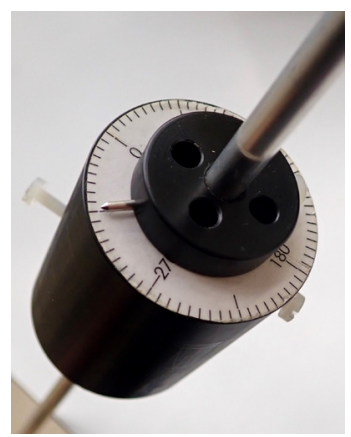

(d)

Figure 3. (a) Top view scheme of the CROCUS reactor core and positions for the installation of the fiber detector for azimuthal measurements. (b) Experimental setup installed in the periphery (PE) position of CROCUS. (c) Plastic holder for the detector front-end. (d) Goniometric piece for the azimuthal positioning.

Two core locations were selected to perform a first set of highly localized measurements with the miniature detector described in Section 2.1: the North West periphery (PE) and the North West control rod (CR) positions (see Fig. 3(a)). Both positions are along the same radial direction and they were selected because of their vicinity to heterogeneous zones of the reactor. Indeed, the PE position is situated right next to the reflector surrounding the reactor core, while the CR lies close to the water gap between the different fuel elements. A first set of measurements in the two positions was carried out in December 2018 and the results are presented in the following Section 3.

A control rod guide tube with a $1.735 \mathrm{~cm}$ internal diameter, identical to the aluminum fuel cladding of CROCUS, is positioned in the CR position for the standard reactor configuration. The guide tube serves for the insertion of a $\mathrm{B}_{4} \mathrm{C}$ absorber control rod for the reactivity control. When empty, the guide tube represents a useful instrumentation channel since the reactor power level is controlled by adjusting the water level or inserting the other symmetrical control rod. On occurrence the tube can be moved to the PE position and, to maintain the same lattice organization in the core, an additional aluminum tube is then placed in the CR position.

The miniature neutron detector prototype was inserted inside the control rod guide tube placed in one of the two positions, PE or CR. A goniometer was built for the accurate positioning of the detector inside the guide tube. It consists of a plastic holder with four holes around its circumference (see Fig. 3(c)), each of 


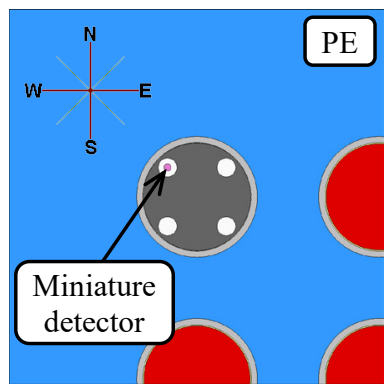

(a)

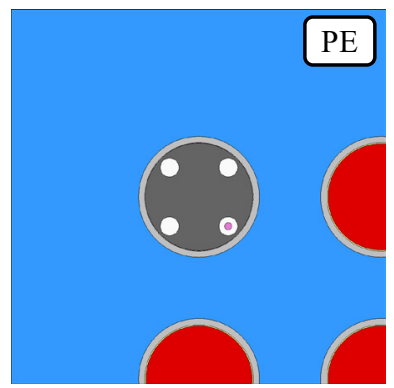

(b)

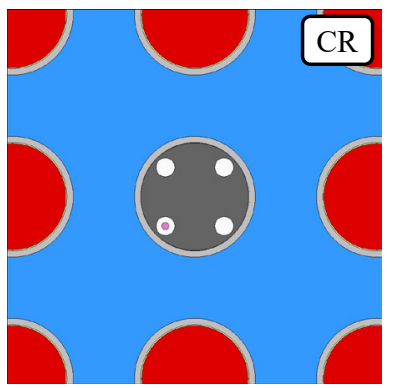

(c)

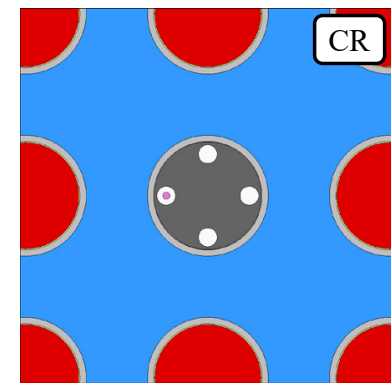

(d)

Figure 4. Examples of experimental measurement arrangements: (a) NW orientation in PE, (b) SE orientation in PE, (c) SW orientation in CR and (d) W orientation in CR.

which accommodates the front end of the miniature detector including the aluminum cap. The hole centers lie within $6.65 \pm 0.01 \mathrm{~mm}$ from the holder central point. The holder is connected to an aluminum bar with a movable plastic element that allows choosing the desired axial location in the core - in this study, $50.0 \pm 0.1 \mathrm{~cm}$ from the bottom of the fuel. The holding piece is equipped with a small metal pin that works as an indication for the azimuthal position of the detector with respect to the scale of the goniometer attached to the guide tube and arbitrarily oriented (in Fig. 3(d)). The experimental setup inside the core is shown in Figure 3(b). The optical fiber was connected to the SiPM placed outside the biological shielding of CROCUS, where also the electronics setup is installed.

The neutron count rates were recorded at four different angular positions in each core location. For the sake of simplicity, these positions are named as their cardinal direction, considering the north direction as represented in Fig. 3(a) and Fig. 4(a). Each measurement was carried out at a reactor power level of approximately $1 \mathrm{~W}$ for 60 and 45 minutes for PE and CR positions, respectively. The number of counts measured with the miniature detector was normalized by the sum of the counts recorded by the two fission chamber monitors. The angular position of the detector, together with the holding device (see Fig.3), has been manually modified after every measurement with the reactor in intermediate conditions (inserted safety blades and water level equal to $800.0 \pm 0.1 \mathrm{~mm}$ ), after waiting for the dose rate inside the reactor cavity to decrease under the allowance limit. It is reasonable to introduce an error of $\pm 2.5^{\circ}$ on the azimuthal positioning of the detector, corresponding to half of the goniometer resolution. Before every measurement the reactor critical state has to be reached and it might not always correspond to the same water level. Variations of $\pm 0.1 \mathrm{~mm}$ from the average water level of $953.5 \pm 0.1 \mathrm{~mm}$ for the PE and $953.3 \pm 0.1 \mathrm{~mm}$ for the CR were observed when azimuthally turning the measuring setup. Although a water level variation will affect the axial profile of the neutron flux, the fluctuations are in the range of the error of the water level gauge and it was demonstrated that a variation of $\pm 0.1 \mathrm{~mm}$ between the periods of a cosine shape is in the order of $10^{-5}$ around the value of $50.0 \pm 0.1 \mathrm{~mm}$. Therefore, it can be concluded that the error on the water level is negligible when estimating the source of uncertainties on the count rate. In a similar way, the uncertainty on the count rate due to the axial positioning of the detector has been estimated to be $0.03 \%$ in the range from 49.9 to $50.1 \mathrm{~cm}$ in the core height. This uncertainty is propagated to the final normalized count rate together with the statistical uncertainty of the neutron detection for both PE and CR positions.

\section{EXPERIMENTAL RESULTS}

The results of the set of measurements performed in the control rod guide tube placed at the core periphery (PE) and at the control rod location (CR) by positioning the miniature detector at the core geometrical mid-height of $50.0 \pm 0.1 \mathrm{~cm}$ are presented in Table I. The number of counts recorded with the miniature detector are normalized by the sum of both the Photonis CFUM21 fission chambers recordings and by the value obtained at the refence angular position chosen to be the NW orientation (corresponding to 0 degrees). 
Table I. Experimental results in periphery position (PE) and control rod position (CR): The fourth column shows the results normalized by the monitor readings and by the value at 0 degrees after the propagation of the error on the detector axial positioning.

\begin{tabular}{|c|c|c|c|}
\hline Position & Orientation & Azimuthal position $\left(^{\circ}\right)$ & $\begin{array}{c}\text { Normalized count rate with total } \\
\text { experimental uncertainty (-) }\end{array}$ \\
\hline \hline PE & NW & $0.0 \pm 2.5$ & $1.0000 \pm 0.0007(0.07 \%)$ \\
\hline PE & NE & $90.0 \pm 2.5$ & $0.9865 \pm 0.0007(0.07 \%)$ \\
\hline PE & SE & $180.0 \pm 2.5$ & $0.9571 \pm 0.0007(0.07 \%)$ \\
\hline PE & SW & $270.0 \pm 2.5$ & $0.9743 \pm 0.0007(0.07 \%)$ \\
\hline \hline CR & NW & $0.0 \pm 2.5$ & $1.0000 \pm 0.0007(0.07 \%)$ \\
\hline CR & SE & $180.0 \pm 2.5$ & $1.0437 \pm 0.0008(0.07 \%)$ \\
\hline CR & SW & $270.0 \pm 2.5$ & $1.0243 \pm 0.0007(0.07 \%)$ \\
\hline CR & W & $315.0 \pm 2.5$ & $1.0038 \pm 0.0007(0.07 \%)$ \\
\hline
\end{tabular}

In addition to the statistical error, the uncertainty on the axial positioning of the scintillator has been quantified and propagated to the final result through normalizations. The final values with their total experimental uncertainty are presented in Table I. The two sets of experimental data are plotted in Fig. 6.

The set of measurements carried out in the PE location shows a minimum towards the SE direction, corresponding to the orientation pointing at the core center. Indeed, a higher share of thermal neutrons is present closer to the core water reflector (the NW direction), where the count rate has its maximum. The gradient in the count rate profile along the radial direction in a range of $1.33 \pm 0.02 \mathrm{~cm}$ has been calculated to be equal to $+(4.29 \pm 0.10) \%$. The positive sign indicates that the gradient is oriented towards increasing radial coordinates, e.g. towards the core boundaries. The specular values at the same coordinate (NE and $\mathrm{SW})$, show a limited gradient of $(1.25 \pm 0.10) \%$ in the NE direction, which might be caused by either unknow experimental biases or by a slightly asymmetry in the CROCUS fuel lattice. Further investigations are needed for determining the source of the NE-SW gradient. In the CR position, a gradient of $-(4.37 \pm 0.10) \%$ is present between the NW and the SE detector positions, meaning that the higher neutron count rate is pointing towards the core center.

\section{COMPARISON WITH PRELIMINARY MONTE-CARLO SIMULATIONS}

The validation of neutron transport simulation with high spatial resolution performed with a Monte Carlo method in full reactor geometries is accompanied by statistical issues. The need for low uncertainties to evaluate effects in the order of few percent difference is worsened due to the small volumes involved in the calculation. For instance, the miniature detector sensitive volume of $0.2 \mathrm{~mm}^{3}$ corresponds to a fraction of $\sim 10^{-10}$ of the total volume of the CROCUS core. Therefore, the computational power required to perform full-core direct Monte Carlo simulations in order to score the ${ }^{6} \mathrm{Li}$ reaction rate at the different azimuthal positions in the real detector volume is prohibitive. However, some approximations can be introduced in the already available Serpent 2 model of CROCUS in order to improve the statistics. The ${ }^{6} \mathrm{Li}$ reaction rate can be scored virtually due to the small self-shielding effect introduced by such a miniature detector. The modelling of the $0.2 \mathrm{~mm}^{3} \mathrm{ZnS}:{ }^{6} \mathrm{LiF}$ material in the geometry would induce a self-shielding effect of approximately $-5 \%$ of the unperturbed neutron flux, which can be neglected if relative results want to be obtained. The use of virtual detector tallies requires a simplification of the holder geometry and allows also to avoid multiple simulations corresponding to the different orientations of the detector. The simplified 
holder is introducing a perturbation of an additional $-5 \%$ of the total neutron flux, which is constant and thus negligible in relative estimations. The interactions can also be scored on a taller virtual volume and, only if absolute estimations want to be performed, the results must be corrected by a form factor given by the averaging of the neutron flux over the axial smearing. Nevertheless, despite these the approximations, it has been estimated that it is possible to reach an uncertainty of $1 \%$ on the normalized ${ }^{6} \mathrm{Li}$ reaction rate in a volume 100 times bigger that the actual detector size after approximately 3300 CPU hours. Most of this computational power is spent in tracking neutrons that have a very low probability to contribute to the 0.2 $\mathrm{mm}^{3}$ detector tally. Therefore, a new iterative approach has been tested in this work with the intent to reduce the uncertainties on the azimuthal reaction rate trend for the PE and CR positions. The approach consists in performing three-steps Serpent 2 simulations going from full-core criticality to source-mode simulation with reduced geometries focusing progressively around the location of interest in the core. After the first two steps, source files for spheres located around the location of interest are written and then used as source input for the successive calculation where the outside geometry is cut out in order to conserve the neutron flux in the selected sphere. After the third step, the azimuthal dependency of the ${ }^{6} \mathrm{Li}$ reaction rate in the CR or PE position is recorded with the same smeared virtual detector tallies described before. The three steps are then repeated multiple times. The results of the virtual detector scores from a series of independent three-steps simulations are averaged to reduce the biasing induced by the two source samplings, assuming that the data obtained are normally distributed. A visual representation of the three-steps approach is shown in Fig. 5. With the iterative three-steps approach it is possible to reach a standard deviation of the results equal to $1.4 \%$ (see Fig. 6 ) on the same smeared volume in approximately 250 CPU hours.

The results obtained with the three-steps method iterated 20 times, normalized by their value at the first azimuthal position of zero degree, are compared with the normalized and adjusted experimental results given in Table I for the PE and CR position. The direct comparison illustrated in Fig. 6 shows a good

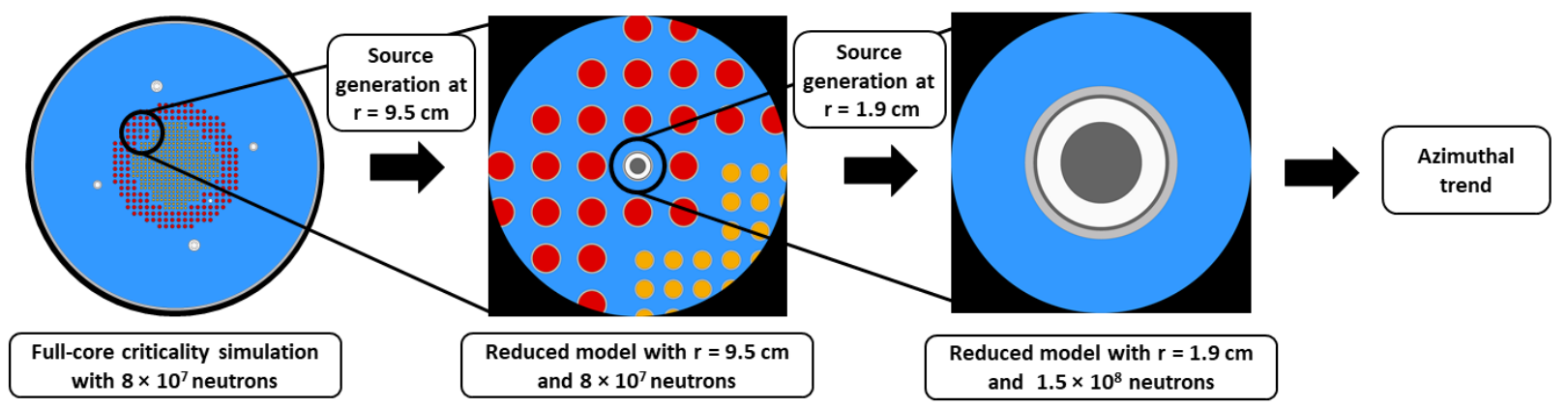

Figure 5. Schematic of the three-steps approach for the computation of the azimuthal trend in the CR position. The three-steps are iterated. The same methodology is used for the PE position.

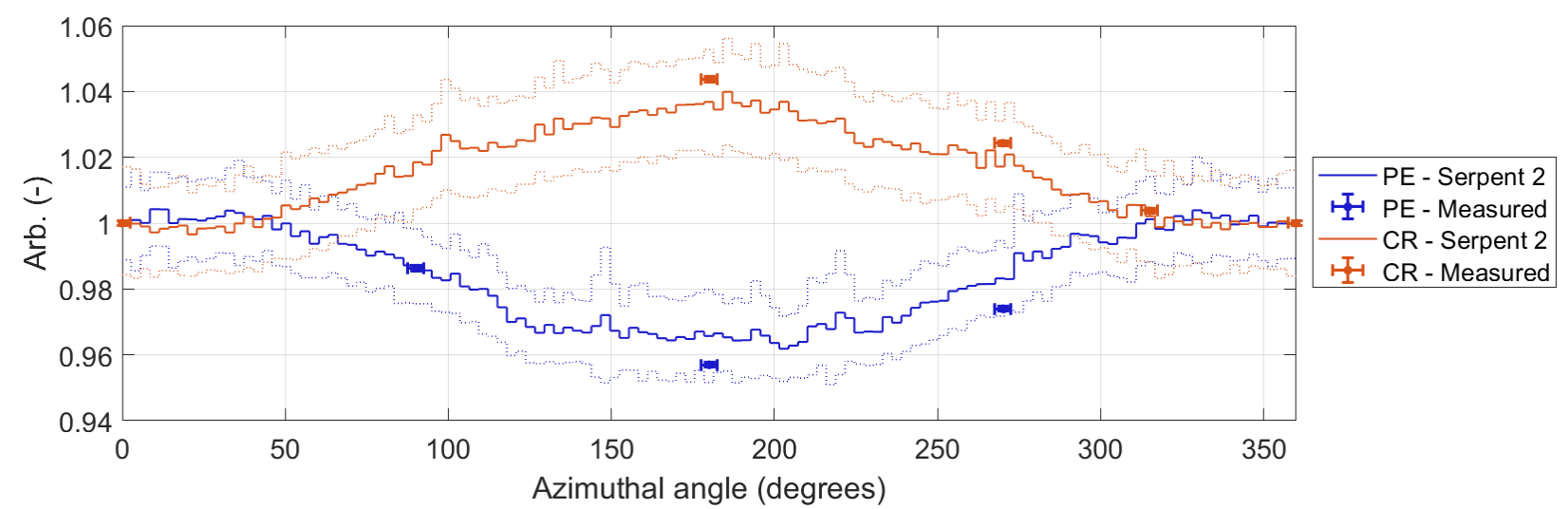

Figure 6. Results for the measured and simulated azimuthal profiles for PE and CR positions. 
agreement between the measurements and the estimation with Serpent 2. All the experimental points lie within the uncertainty of the simulated results, being of $1.2 \%$ and $1.4 \%$ on average for PE and CR, respectively. At the same time, the good efficiency of the iterative three-steps method suggests that the same reaction rate could be scored on volumes that are shorter in the axial direction without the need for prohibitive computational power.

\section{CONCLUSIONS}

A miniature neutron detector with a sensitive volume of $0.2 \mathrm{~mm}^{3}$ has been used to measure with a mm-precision the azimuthal count rate profile within a control rod guide tube of the zero-power CROCUS reactor positioned at two different locations of interest: in the core periphery and in the control rod position. The two sets of experiments presented in this work proved the feasibility of performing highly spatiallyresolved measurements in CROCUS and show the possibility to resolve count rate gradients of few percent within the $\mathrm{cm}$ range. A gradient of $+(4.29 \pm 0.10) \%$ with the control rod guide tube diameter of $1.3 \mathrm{~cm}$ has been measured close to the water reflector. The gradient is oriented towards the core boundaries due to the higher share of thermal neutrons. On the contrary, closer to the core center a gradient of $-(4.37 \pm 0.10) \%$ is inward oriented. The experimental values have been compared with the results of Monte Carlo simulations, performed by using an iterative three-steps method with Serpent 2. All the measurement points are in the range of uncertainty of the calculated azimuthal trend of the ${ }^{6} \mathrm{Li}$ reaction rate. In conclusion, this work paves the way for the investigation of localized phenomena in the CROCUS core and for the use of the experimental results with high spatial resolution for the validation of high-fidelity neutronics codes.

\section{REFERENCES}

1. International Atomic Energy Agency, Operation and Licensing of Mixed Cores in Water Cooled Reactors, IAEA-TECDOC-1720, IAEA, Vienna (2013). [Online]: https://wwwpub.iaea.org/MTCD/Publications/PDF/TE-1720 web.pdf

2. Westinghouse, TRITON11TM - Westinghouse 11x11 BWR fuel design. [Online]: http://www.westinghousenuclear.com/Portals/0/Triton\%20A4 v7.pdf

3. K. Macku, F. Jatuff, M. Murphy, M. Plaschy, P. Grimm, O. P. Joneja and R. Chawla, "Radial and Azimuthal ${ }^{235} \mathrm{U}$ Fission and ${ }^{238} \mathrm{U}$ Capture Distributions in BWR $\mathrm{UO}_{2}$ Pins: CASMO-4 and MCNP4C versus Activation Foil Measurements," Nuclear Science and Engineering, 155:1, pp. 96-101 (2007).

4. F. Vitullo, V. Lamirand, A. Pautz, "A novel neutron detector for localized in-core measurements in the CROCUS reactor for high-fidelity code validation purposes", in Poster session at the FISA2019 conference, 4-7 June 2019, Pitesti, Romania.

5. V. Lamirand, A. Rais, S. Hübner, O. Pakari, M. Hursin, A. Laureau, C. Lange, J. Pohlus, U. Paquee, C. Pohl, P. Frajtag, D. Godat, G. Perret, C. Fiorina, and A. Pautz, "First results of neutron noise experiments in the AKR-2 and CROCUS reactors for the European project CORTEX," submitted to IEEE Transactions on Nuclear Science on October $1^{\text {st }}, 2019$.

6. F. Vitullo, V. Lamirand, J.-B. Mosset, P. Frajtag, O. Pakari, G. Perret and A. Pautz, "Developing and testing a miniature fiber-coupled scintillator for in-core neutron counting in CROCUS," in Proceedings of International conference on Advancements in Nuclear Instrumentation Measurement Methods and their Applications (ANIMMA2019), Portorož, Slovenia, 17-21 June 2019.

7. F. Vitullo, V. Lamirand, J.-B. Mosset, P. Frajtag, O. Pakari, G. Perret and A. Pautz, "A $\mathrm{mm}^{3}$ fibercoupled scintillator for in-core thermal neutron detection in CROCUS," submitted to IEEE Transactions on Nuclear Science on October $1^{\text {st }}, 2019$.

8. V. Lamirand, Installation nucléaire CROCUS - Rapport de sécurité version 1.2, Lausanne (2017). 